\title{
An Improved High-Precision Calibration Procedure For Reference Standard Hydrometers
}

\author{
Horace A. Bowman and William H. Gallagher
}

Institute for Basic Standards, National Bureau of Standards, Washington, D.C. 20234

(June 24, 1969)

\begin{abstract}
This paper describes a method of calibrating hydrometers which, although no more accurate that the technique used heretofore at the National Bureau of Standards, reduces calibration time from several days to a few hours. The procedure is based upon easily measured hydrometer weight, stem scale geometry, and flotation level measurements at only one liquid density. The reliability of this single level is improved by observing the hydrometer flotation under various weight loads. The calibration experiment is oriented toward automatic data reduction so the finished calibration report is of much wider applicability than the simple correction table derived from the older experiment.

Key words: Calibration; density; hydrometer; hydrometer calibration; reference hydrometer; specific gravity; standard hydrometer.
\end{abstract}

\section{Objectives and Results}

There are two classes of hydrometers, which although identical in construction, differ significantly in their method of calibration and the use to which they are put. Working hydrometers are used extensively in science and industry for moderate precision measurements of liquid densities. Reference standard hydrometers are used exclusively for the calibration of working hydrometers using comparison techniques described in the appendix. These reference standard hydrometers are calibrated by comparing their observed flotation levels to bath densities independently determined by hydrostatically weighing solid object density standards.

The comparison experiment used in the past at NBS for calibration of reference standard hydrometers was fundamentally sound under specified conditions, but it was time-consuming and its range of applicability was restricted. Hydrometers were tested in the liquid in which they were to be used, and several baths of this liquid were prepared covering the density range shown on the hydrometer stem scale. It was difficult to obtain more than five such comparisons in a day, although the requirements of some calibrations were such that fifteen or more comparisons had to be performed. Additionally, if the hydrometer was to be used in a liquid of different surface tension, a different calibration was required.

We have devised a calibration procedure in which only one comparison bath is required which can be completed in less than three hours, and the data are of such form that corrections can be deduced for any (or all) points on the stem scale. Even though the hydrometer is to be used in fluids of radically different surface tension or temperature, additional calibration effort is not required.

This new procedure is based upon knowledge of the hydrometer weight and stem scale geometry (both easily measured to adequate accuracy) and knowledge of the level at which the hydrometer floats in one bath of known properties. Hydrometer flotation levels are observed to one-tenth scale division. In estimating tenths of divisions, most observers are biased for or against certain numbers, and when reading the same level repeatedly, they will usually continue to make the same estimate. As a result, multiple observations on the same level are little better than a single observation. To minimize such reading errors, only one observation is made on the freely floating hydrometer. Thereafter it is loaded by small weights, causing it to sink to lower levels, each of which is recorded. From knowledge of the weights and stem scale geometry it is possible to calculate independent values of the unloaded flotation level. Averaging the single observed value with the various calculated values provides a more reliable figure for the true flotation level of the unloaded hydrometer in the bath. This bath may be set up to have ideal properties such as transparency, surface tension, or any other characteristic considered important by the experimenter. The requirement is that it float the hydrometer near the lower end of its scale.

Based upon long standing custom the densities of different classes of fluids are measured on different scales of density; for example, oils are usually measured in "degrees API," alcohol in "percent of proof 
spirit," and acids in "specific gravity." This has forced NBS to maintain an inventory of currently calibrated reference standard hydrometers whose scales are graduated in different systems but covering the same density range. Our new calibration procedure is oriented for automatic data reduction. This yields a byproduct. With appropriate computer instructions, we are able to obtain a table of scale corrections to convert readings into many other density scales without additional laboratory work, so a single calibration experiment of the type described can take the place of several of the older experiments.

The National Physical Laboratory $[1]^{1}$ calibrates its reference hydrometers by partially suspending them from a balance while floating in a xylene bath at some previously selected scale graduation. The bath density is measured by a special finely divided hydrometer. By varying the suspension length and the xylene level so that each cardinal point is examined, comparison between gravitational and buoyant forces provides the required correction.

\section{The Calibration Experiment}

There are four steps in the laboratory work demanded by the new procedure. As pointed out above, about three hours is required for completion.

1. The hydrometer is weighed on a laboratory balance to determine its apparent weight in air, $\bar{W}_{H}$, defined and discussed in section 4 .

2. The hydrometer stem scale of $N$ cardinal points is laid alongside a centimeter scale (actually a linear comparator, see fig. 1) and a table of values of $L_{1}, L_{2}$, $L_{3}, \ldots L_{N}$ is prepared indicating the distance of each cardinal point from some arbitrary zero reference. At the same time the distance to the upper tip of the hydrometer, $L_{T}$, is recorded.

3. An indicator micrometer, see figure 1 , is used to measure the stem diameter at each cardinal point. Two measurements, $90^{\circ}$ apart, are averaged and a table of values $D_{1}, D_{2}, D_{3} \ldots D_{N}$ is prepared showing these average stem diameters.

4. The hydrometer is floated in a bath of density $\rho$, which is measured by hydrostatically weighing a solidobject density standard as discussed in section 4 . The hydrometer flotation level is noted. After this, four (or more) hat-shaped objects of weights in air $\bar{W}_{A}, \bar{W}_{B}$, $\bar{W}_{C}$, and $\bar{W}_{D}$ are placed on the upper tip of the hydrometer, causing it to sink deeper into the bath. The flotation level with each hat in place is noted.

\section{Data Recording and Reduction}

Raw data are recorded on forms designed so that a typist can most conveniently transcribe them on a punched tape acceptable to the NBS ADP system and receive the finished calibration report from the printout. This requires about six minutes.

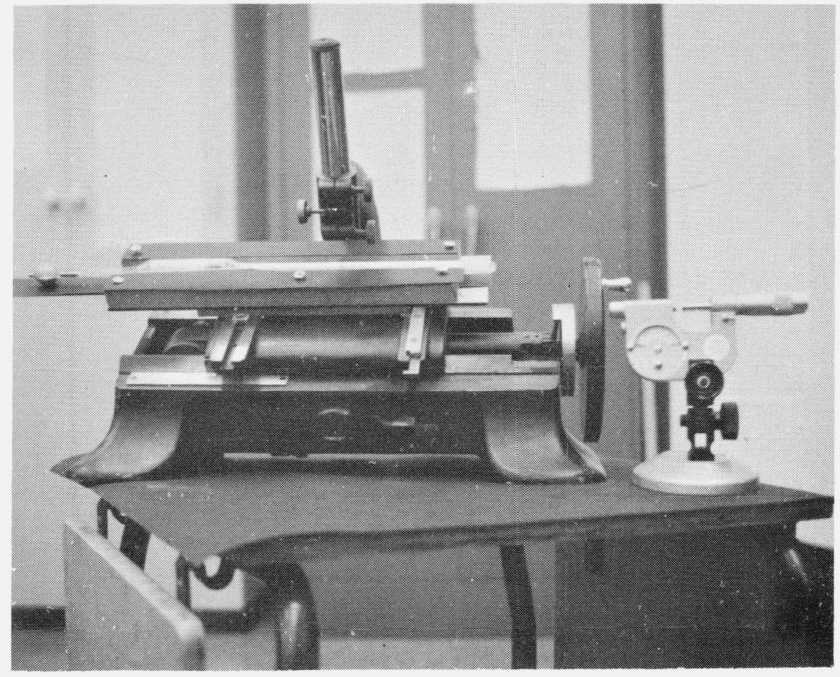

Figure 1. The two devices used to measure hydrometer stem geometry.

The linear comparator on the left is used to convert the stem scale graduations from density indications to linear distances. Stem diameters are measured by the indicator micrometer shown on the right.

The computer program is based upon the following arguments:

1. The five equilibrium flotation levels observed during the flotation tests (sec. 2.4) are observed on the hydrometer stem scale in terms of the scale units. These levels must be expressed in ordinary length units, centimeters. This is accomplished by interpolation in the table of centimeter equivalents prepared in section 2.2, after which we have $L_{0}, L_{A}, L_{B}, L_{c}$, and $L_{D}$ representing the level in centimeters at which hydrometer flotation occurs with no hat, with hat $A$, with hat $B$, with hat $C$, etc. If the bath temperature is different from the room temperature during geometric measurements, then the geometric measurements are corrected to the bath temperature by suitable application of the thermal coefficient of expansion of the hydrometer.

2. By similar interpolation we obtain from the table of stem diameters (sec. 2.3) the stem diameter at each flotation level. Values of $D_{0}, D_{A}, D_{B}, D_{C}$, and $D_{D}$ are the stem diameters at each of the five flotation levels no hat, with hat $A$, with hat $B$, with hat $C$, etc.

3 . We now calculate the average stem diameter between the "no hat" flotation level and each of the "with hat" levels. Values of $\bar{D}_{A}, \bar{D}_{B}, \bar{D}_{C}, \bar{D}_{D}$ are the average stem diameter between $L_{0}$ and $L_{A}$, between $L_{0}$ and $L_{B 0}$ between $L_{0}$ and $L_{C}$, and between $L_{0}$ and $L_{D}$.

4. From knowledge of the surface tension of the test bath, $\gamma$, and the various stem diameters involved, we calculate the weight in air of the meniscus, $m$, which would form at each flotation level and cardinal point, thus

$$
m=\frac{\pi D \gamma}{980}
$$


where $D$ is the stem diameter at the level of interest. ${ }^{2}$ Meniscus weights at the various flotation levels are named $m_{0}, m_{A}, m_{B}, m_{C}$, and $m_{D}$ depending upon the hat in use. At the cardinal points, they are labeled $m_{1}, m_{2}, m_{3} \ldots m_{N}$.

5. The standard deviation of a single observation on the flotation level of a hydrometer may be as large as $1 / 4 \mathrm{~mm}$ when observing conditions are non-ideal. Step 2.4 in the experimental procedure yields only one value of the flotation level, $L_{0}$, of the hydrometer (by itself with no hat load) in the test bath. The objections to repeated observations of the same stem scale graduation to improve assurance were discussed above. We calculate an additional value of this flotation level from each of the "with hat" flotation level observations. These additional values are independent of the original observed value, and they are largely, but not entirely, independent of one another.

The flotation level under no load is taken under the following conditions of equilibrium, see figure 2:

$$
M+m_{0}-\rho V_{0}-\rho_{A}\left(V-V_{0}\right)=0
$$

where $M$ and $V$ are the mass and volume of the hydrometer, $\rho_{A}$ the air density, and $V_{0}$ is the immersed volume. The comparable equation of equilibrium for flotation when the hydrometer is loaded by hat " $A$ " (with the hydrometer floating at stem level $L_{A}$ ) is given by

$$
M+\bar{W}_{A}+m_{A}-\rho V_{0}-\rho V_{A}-\rho_{A}\left(V-V_{0}-V_{A}\right)=0
$$

where $V_{A}$ is the stem volume between $L_{0}$ and $L_{A}$. Subtracting eq (2) from (1) we get a value for $V_{A}$

$$
V_{A}=\frac{\bar{W}_{A}+m_{A}-m_{0}}{\rho-\rho_{A}} .
$$

Based upon stem geometry we can write a different equation for $V_{A}$

$$
V_{A}=\frac{\pi \bar{D}_{A}^{2}}{4}\left(L_{0}-L_{A}\right)
$$

where $\bar{D}_{A}$ is the average stem diameter between $L_{0}$ and $L_{A}$. Equating (3) and (4) yields a new value of $L_{0}$, thus

$$
L_{0}=L_{A}-\frac{4\left(\bar{W}_{A}+m_{A}-m_{0}\right)}{\pi \bar{D}_{A}^{2}\left(\rho-\rho_{A}\right)} .
$$

Using this procedure, we calculate a value of $L_{0}$ for each "with hat" observation and take the average to give the reference level, $L$; thus

${ }^{2}$ If $\gamma$ is a pure force, then $m$ as defined here would be mass, not weight in air. However, air buoyancy is ignored in most surface tension measurements, so $\gamma$ is usually an apparent force. If $\gamma$ is a pure force, the meniscus weight in air is

$$
\frac{\pi D \gamma}{980}\left[1-\frac{\rho_{A}}{\rho}\right]
$$

where $\rho_{A}$ is air density and $\rho$ the fluid density. For the average hydrometer, the difference between meniscus weight and mass is about $1 \mathrm{ppm}$ of the hydrometer weight -insignificant in this experiment.

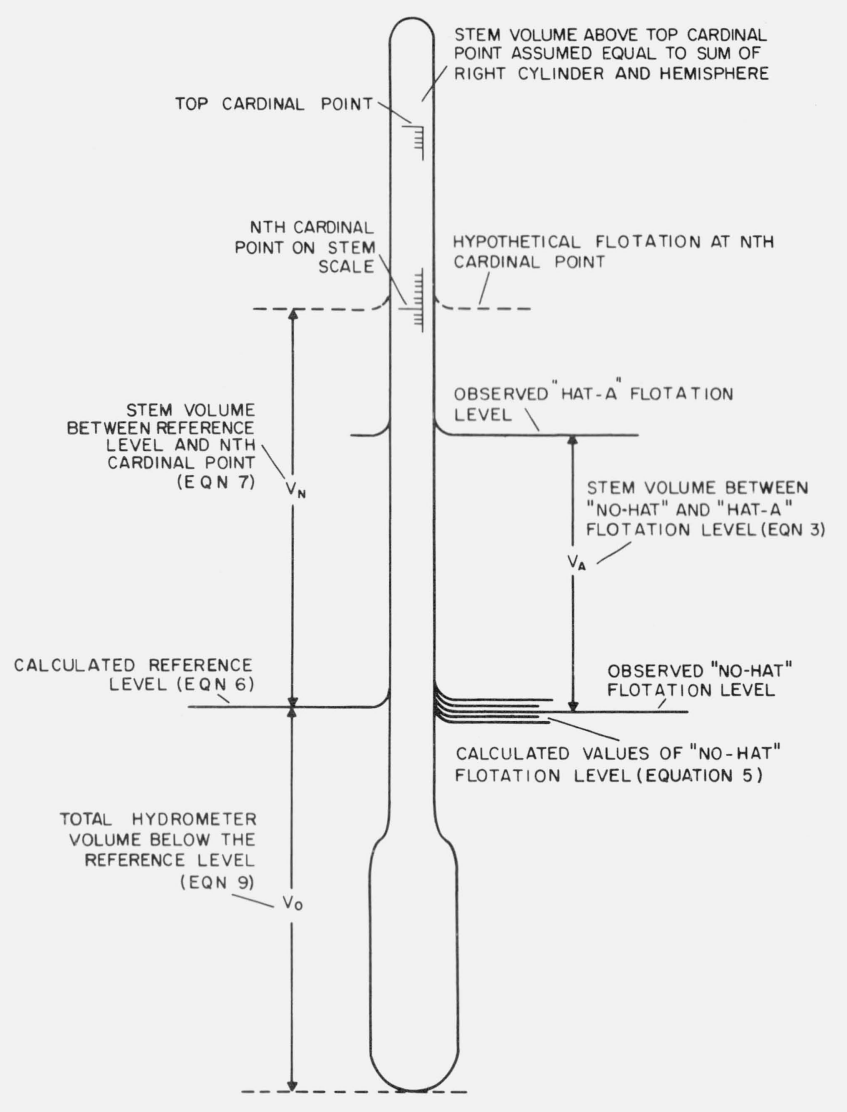

FIGURE 2. Various stem scale levels and volumes used in the datareduction argument.

$L=\frac{L_{0}(\mathrm{obs})+L_{0}(\text { hat } A)+L_{0}(\text { hat } B)+L_{0}(\text { hat } C)+L_{0}(\text { hat } D)}{5}$.

The variability in these values of $L_{0}$ is mostly random and is associated with imperfect flotation and geometric observations.

6 . We next calculate a table of stem volumes between the reference level, $L$, and each cardinal point on the stem scale. Values of $V_{1}, V_{2}, V_{3} \ldots V_{N}$ are obtained from

$$
V_{N}=\frac{\pi \bar{D}_{N}^{2}}{4}\left(L-L_{N}\right)
$$

where $\bar{D}_{N}$ is the average stem diameter between $L$ and $L_{N}$.

7. Based upon our knowledge that the hydrometer floats at the reference level, $L$, under "no hat" conditions when test bath temperature, density and surface tension are $t, \rho$, and $\gamma$ respectively, we can calculate a table of bath densities $\rho_{1}, \rho_{2}, \rho_{3}, \rho_{4} \ldots \rho_{N}$ which will float the hydrometer at each of the $N$ cardinal points under conditions of $t$ and $\gamma$. 
Equilibrium conditions for flotation at the reference level, $L$, are given by

$$
m_{0}+\left(M-\rho_{A} V\right)-\rho V_{0}+\rho_{A} V_{0}=0
$$

where $V_{0}$ is the hydrometer volume below $L$. As explained in section 4 , the apparent weight in air of the hydrometer, $\bar{W}_{H}$, is given by $M-\rho_{A} V$. Making this substitution and solving for $V_{0}$ we get

$$
V_{0}=\frac{\bar{W}_{H}+m_{0}}{\rho-\rho_{A}} .
$$

By similar reasoning we can write the equilibrium equation for flotation at $L_{N}$ (the level of the $N$ th cardinal point)

$$
\begin{aligned}
& M+m_{N}-\rho_{N}\left(V_{0}+V_{N}\right)-\rho_{A}\left(V-V_{0}-V_{N}\right)=0 \\
& \bar{W}_{H}+m_{N}-\rho_{N}\left(V_{0}+V_{N}\right)+\rho_{A}\left(V_{0}+V_{N}\right)=0 .
\end{aligned}
$$

The immersed volume, $V_{i}$, is the sum of $V_{0}$ and $V_{N}$. Looking to eqs (7) and (9) for numerical values of $V_{0}$ and $V_{N}$, we get

$$
V_{i}=V_{0}+V_{N}=\frac{\bar{W}_{H}+m_{0}}{\rho-\rho_{A}}+\frac{\pi \bar{D}_{N}^{2}}{4}\left(L-L_{N}\right) .
$$

All terms in (14) represent vertical forces on the hydrometer, positive down. Inasmuch as we want the hydrometer to float at the same level (namely the $N$ th cardinal point) under both initial and final conditions, the sum of terms involving $\Delta$ (after expansion of (14)) must be held to zero.

Expanding (14) and subtracting (13) leaves

$$
\Delta m_{N}-\rho_{N} \Delta V_{i}-\Delta \rho_{N} V_{i}-\Delta \rho_{N} \Delta V_{i}-\rho_{A} \Delta V_{e}=0
$$

and

$$
\Delta \rho_{N}=-\frac{\rho_{N} \Delta V_{i}+\rho_{A} \Delta V_{e}-\Delta m_{N}}{V_{i}+\Delta V_{i}} .
$$

Inasmuch as volumes are functions of temperature, and meniscus weight is a function of surface tension

$$
\begin{aligned}
\Delta V_{i} & =\alpha(T-t) V_{i}=\alpha(T-t)\left(V_{0}+V_{N}\right) \\
\Delta V_{e} & =\alpha(T-t) V_{e}=\alpha(T-t)\left(V-V_{0}-V_{N}\right) \\
\Delta m_{N} & =\frac{\pi D_{N}}{980}(\Gamma-\gamma)
\end{aligned}
$$

where $\alpha$ is the volumetric coefficient of expansion of the hydrometer. Substituting values of the $\Delta$ terms in (17) back into (16) we get

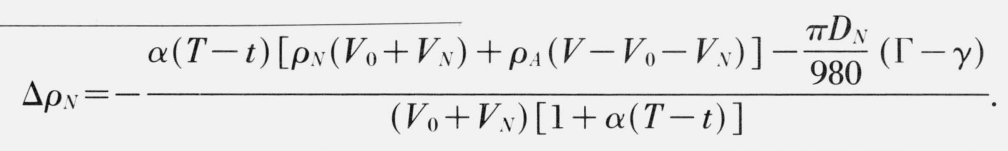

With a numerical value of $V_{i}$ from (11) we solve (10B) for $\rho_{N}$

$$
\rho_{N}=\frac{\bar{W}_{H}+m_{N}+\rho_{A} V_{i}}{V_{i}} .
$$

8. Values of $\rho_{1}, \rho_{2}, \rho_{3}, \ldots \rho_{N}$ calculated in eq (12) are valid only when the flotation liquid is identical to the test bath in that it has the same temperature, $t$, and the same surface tension, $\gamma$. We must, therefore, construct a table of densities which will float the hydrometer at each of the $N$ cardinal points under the general conditions of temperature, $T$, and surface tension, $\Gamma$.

Equation (10A) shows equilibrium conditions at the $N$ th cardinal point under $t$ and $\gamma$. The exposed volume of the hydrometer, $V_{e}$, is given by

$$
V_{e}=V-V_{0}-V_{N} .
$$

Then from (10A)

$$
M+m_{N}-\rho_{N} V_{i}-\rho_{A} V_{e}=0 .
$$

Since altering $t$ and $\gamma$ of the bath will cause changes in $m_{N}, \rho_{N}, V_{i}$, and $V_{e}$, eq (13) becomes

$$
\begin{aligned}
M+\left(m_{N}+\Delta m_{N}\right)-\left(\rho_{N}+\Delta \rho_{N}\right)\left(V_{i}+\Delta V_{i}\right) & \\
& -\rho_{A}\left(V_{e}+\Delta V_{e}\right)=0 .
\end{aligned}
$$

From (10A) the bracketed term of the numerator of (18) is seen to be equal to $\left(M+m_{N}\right)$, so that

$$
\Delta \rho_{N}=-\frac{\alpha(T-t)\left(M+m_{N}\right)-\frac{\pi D_{N}}{980}(\Gamma-\gamma)}{V_{i}[1+\alpha(T-t)]} .
$$

After selecting values for $T$ and $\Gamma$, all terms on the right side of (19) are known, ${ }^{3}$ and

$$
R_{N}=\rho_{N}+\Delta \rho_{N}
$$

where $R_{Y}$ is the liquid density which will float the hydrometer at its $N$ th cardinal point when the liquid temperature is $T$ and its surface tension is $\Gamma$.

If geometric measurements associated with this test are made in centimeters, air and sinker densities in grams per cubic centimeter, surface tensions in dynes per centimeter and weighings in grams, then all terms eq (20) will be in grams per cubic centimeter. If $S_{N}$ is the numerical value engraved alongside the $N$ th cardinal point on the stem scale of the hydrometer, then

$$
C_{N}=R_{N}-S_{n}
$$

${ }^{3}$ The procedure for obtaining a value of $M$ in eq (19) from the balance observation is explained in section 4 . 
where $C_{N}$ is the correction which will convert the $N$ th cardinal point into grams per cubic centimeter, provided that (a) the fluid in which the hydrometer floats is at temperature $T$ and (b) has a surface tension of $\Gamma$. Violation of either condition will result in false values in eq (21).

Inasmuch as the ADP equipment is easily instructed to calculate tables of values of $\Delta \rho_{N}$, eq (19), for a wide selection of $T$ 's and $\Gamma$ 's, a correction table which is appropriate to any foreseeable set of conditions is conveniently available.

\section{Use of The Balance}

Observation of the hydrometer floatation level to $0.1 \mathrm{~mm}$ requires an experienced observer working under near-ideal circumstance. Such an observation represents a precision of only 5 in $10^{4}$ of a typical $20-\mathrm{cm}$ stem scale. In order to reduce calibration effort without degrading quality, we have eliminated from the calibration procedure most of these difficult and relatively low-precision measurements, and substituted in their place measurements of other hydrometer properties which can be made to a much higher order of precision with only a fraction of the effort.

The new calibration procedure relies strongly upon weight calculations based on balance observations. On modern single-pan direct-reading balances such determinations are easily made to parts per million. During the calibration it is necessary to determine the apparent weight in air of the hydrometer, $\bar{W}_{H}$, for use in eq (9), and it is also necessary to determine the apparent weight of the solid-object density standard while immersed in the test bath for calculation of $\rho$ as explained below.

There are two other tasks which are easily performed on such balances which, although important to the hydrometer calibration, are not a part of the calibration procedure. The first task is the determination of the mass of the hats used in the calibration, and the second is the determination of the mass and volume of the solid-object density standard.

Bowman and Schoonover [2] have studied the performance of single-pan direct-reading balances in the above mentioned applications. The brief comments to follow are justified in their paper.

The apparent weight of an object in a medium is a force which is defined as the difference between the downward mass force exerted by the object and the upward buoyant force exerted on the object by the medium. Thus, in eq (9), the apparent weight in air of the hydrometer, $\bar{W}_{H}$, is given by

$$
\bar{W}_{H}=M-\rho_{A} V
$$

where $M$ and $V$ are the mass and volume respectively of the hydrometer, and $\rho_{A}$ the air density at the instant of observation. Since air density is a parameter which is constantly changing with variations in barometric pressure, the temperature of the air, and its relative humidity (in that order of significance), it is necessary to measure these quantities and calculate $\rho A$ from

$$
\rho_{A}=\frac{.464554 B_{A}-H_{A}\left(.00252 T_{A}-.020582\right)}{T_{A}+273.16}
$$

where $B_{A}, T_{A}$, and $H_{A}$ are the measured values of barometric pressure (in $\mathrm{mm}$ of mercury), temperature of the air (in ${ }^{\circ} \mathrm{C}$ ), and relative humidity (in percent).

$\bar{W}_{H}$ is the force exerted on the balance pan by the hydrometer: however, for reasons explained in the Bowman-Schoonover paper, the balance observation, $O_{H}$, must be corrected to obtain $\bar{W}_{H}$

$$
\bar{W}_{H}=\left(O_{H}+C_{1}\right)\left(1-\frac{\rho_{A}}{\rho_{\mathrm{s}}}\right)
$$

where $C_{1}$ is the true mass correction to the balance reading and $\rho_{s}$ is the density of the built-in balance weights. $C_{1}$ and $\rho_{s}$ may be obtained from the manufacturer, or the experimenter may obtain himself by techniques described by Mrs. Mildred Jones in the appendix to the above paper. The numerical value of $W_{H}$ obtained from eq (23) is the value used in eq (9) in the basic data reduction.

The numerical value of $M$ required in eq (19) may be obtained from this value of $W_{H}$ and an estimate of $V$, and since $M=\bar{W}_{H}+\rho_{A} V . V$ is adequately approximated by assuming that the tip volume of the hydrometer stem (fig. 2) is equal to the sum of a right circular cylinder and a hemisphere; thus

$$
V=\left[\left(L_{T}-L_{N}-\frac{D_{N}}{2}\right)\left(\frac{\pi D_{N}^{2}}{4}\right)+\frac{1}{12} \pi D_{N}^{3}\right]+V_{0}+V_{N}
$$

where the bracketed term is the tip volume. For minimum error in this approximation, $\mathrm{D}_{N}, L_{N}$, and $V_{N}$ should be taken to refer to the top cardinal point on the stem scale.

If we know the mass and volume of an object by virtue of an independent experiment, we can combine this knowledge with a balance-determination of the object's apparent weight in a medium to obtain the density of the medium itself. This is the technique used to determine the density of the test bath, $\rho$, in eq (1). By a previous hydrostatic and air weighing we determine the mass, $M_{s}$, and volume, $V_{s}$, of the solid-object density standard. We now suspend the density standard in the test bath by a fine wire from the balance pan. From the foregoing arguments we know that the apparent weight of the density standard in its medium (that is, the test bath) is

$$
\bar{W}_{s}=M_{s}-\rho V_{s}
$$

where $\rho$ is the bath density. Also, from (23), we know

$$
\bar{W}_{s}=\left(O_{s}+C_{2}\right)\left(1-\frac{\rho_{\mathrm{A}}}{\rho_{\mathrm{s}}}\right)
$$

where $C_{2}$ is the true mass correction to the balance 
reading, $O_{s}$. Equating the above two expressions for $\bar{W}_{s}$ and solving for $\rho$, we get

$$
\rho=\frac{M_{s}-\left(O_{s}+C_{2}\right)\left(1-\rho_{\mathrm{A}} / \rho_{\mathrm{s}}\right)}{V_{s}} .
$$

Equation (24) provides a numerical value of $\rho$ for use in the basic data reduction format described in the previous section.

\section{The Calibration Report}

Except in very unusual cases, a modern instrument calibration must contain more than a table of scale corrections. It should state as precisely as possible the conditions under which the calibration is valid and also the degree of validity. In addition to density, hydrometers are sensitive to the temperature and surface tension of the liquid in which they float, so all three parameters must be examined. Ignoring these two second-order variables results in a calibration of very limited applicability. Under such circumstances the hydrometer can be used only in liquids whose temperatures and surface tensions are equal to those of the test bath. Eq (17) introduce our best estimates of these extraneous effects into the calibration.

One sheet of the calibration printout is shown in figure 3. Each table provides scale corrections to each

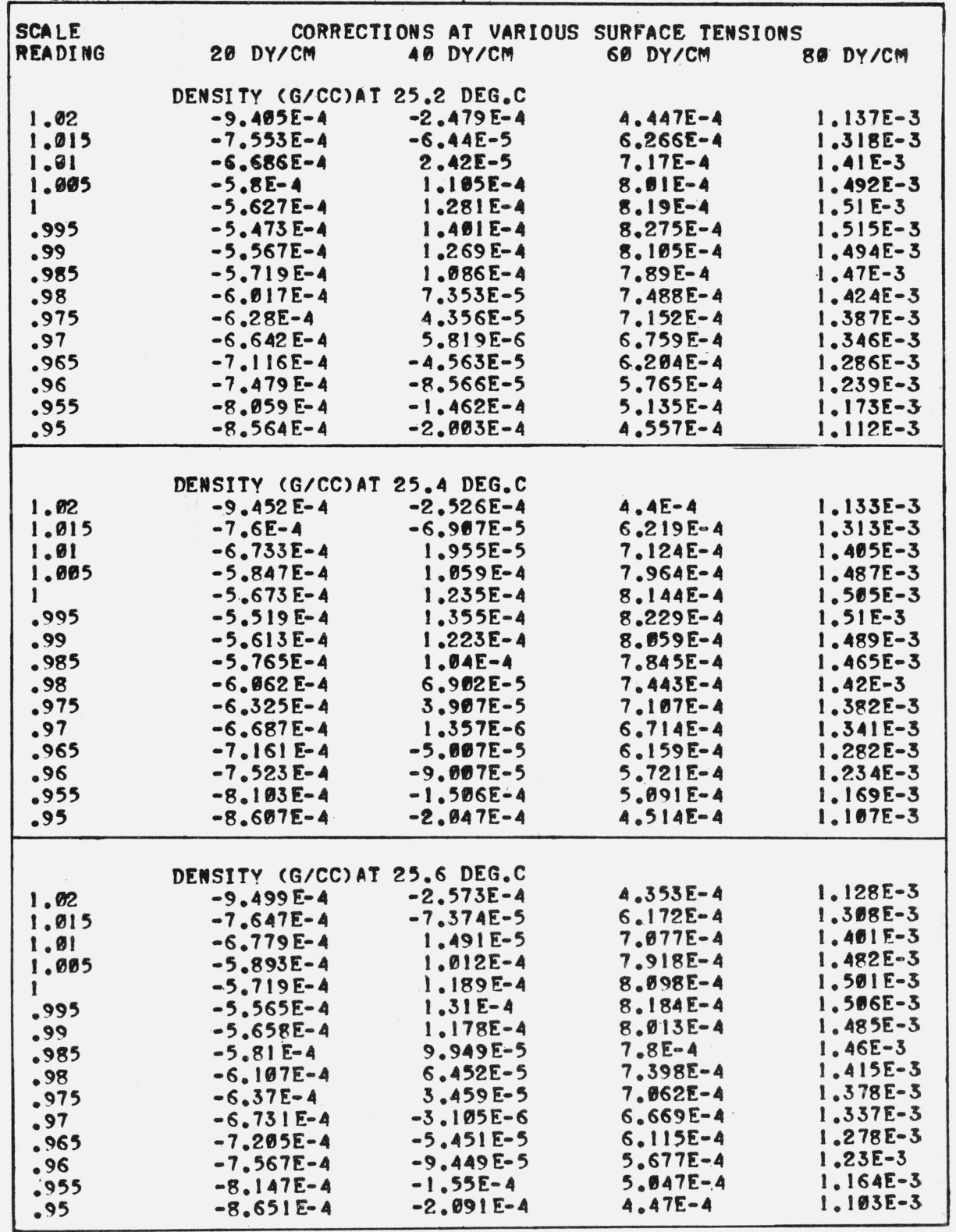

FiguRE 3. One page of a multiple-page complete calibration.

Since hydrometer response to density varies with both temperature and surface tension, a complete hydrometer calibration contains various columns to allow for changes in surface tension, and also it contains different tables for different temperatures. 
cardinal point of the stem scale, reducing the observation to density in grams per cubic centimeter. The table is multi-columned in surface tension from 20 to 80 dynes per centimeter. To account for differences in hydrometer performance as a function of temperature, a separate table is given for each $0.2{ }^{\circ} \mathrm{C}$ over the temperature range for which calibration is required. This type of calibration report has the widest possible utility.

Occasionally a calibration is called for under very restricted conditions. Such a calibration is shown in figure 4 . In this case, the hydrometer is asked to read in terms of one of the numerous specific gravity scales, namely S.G. $\left(25 / 15.556{ }^{\circ} \mathrm{C}\right)$, which is defined as the quotient of the density of the liquid at $25^{\circ} \mathrm{C}$ divided by water density at $15.556^{\circ} \mathrm{C}$. When such a calibration is requested, suitable computer instruction makes $T=25{ }^{\circ} \mathrm{C}$ and calculated densities divided by suitable water density values in the various data reduction equations. Again the calibration is multi-columned in surface tension inasmuch as the hydrometer user can rarely predict the surface tension of the liquid in which the hydrometer is to be used. As will be pointed out in the appendix, this may be a very unstable factor.

There are two classes of errors in the hydrometer calibration. The systematic errors associated with the test apparatus are assumed to be about equal in all NBS calibrations of this type. We can obtain an estimate of these errors by noting the change in the computed corrections when the various input data items are varied at the computer input by the estimated uncertainty in each. Table 1 shows that the effect of these errors is about 0.00005 S.G.

As previously explained, we believe the random errors in the calibration to be fairly well indicated by the scatter of the five values of $L$ in eq (6). For the calibration shown in figure 4 , this amounts to a standard deviation of 0.000075 S.G.

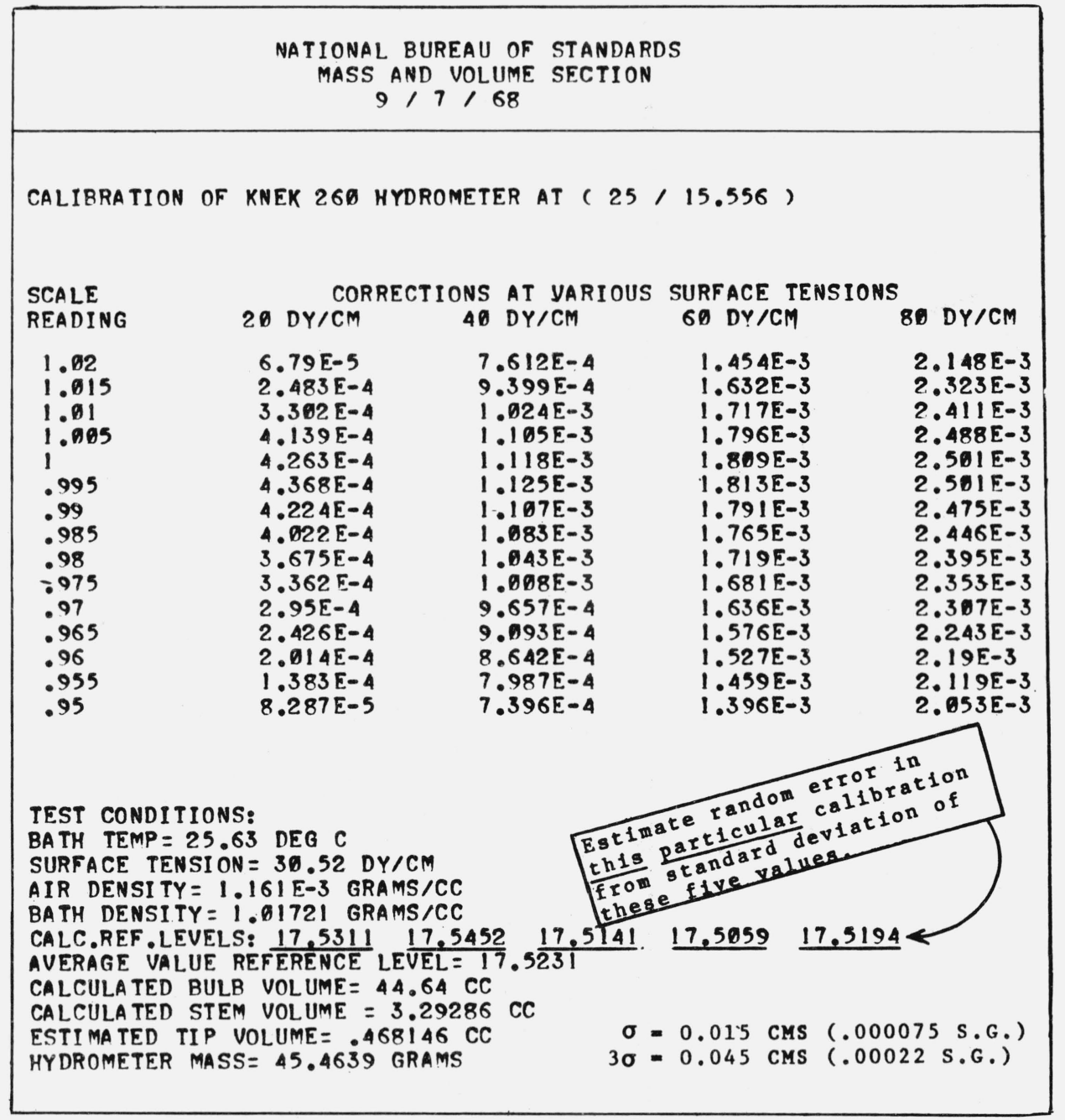

Figure 4. Calibration $\left(25 / 15.556{ }^{\circ} \mathrm{C}\right)$, a specific gravity scale which is valid only at $25^{\circ} \mathrm{C}$.

\footnotetext{
A partial calibration is adequate if the hydrometer will always be used at some known temperature.
} 
TABLE 1 .

\begin{tabular}{|c|c|c|c|}
\hline Input Data Item & $\begin{array}{c}\text { Estimated } \\
\text { uncertainty } \\
\text { in input } \\
\text { data item** }\end{array}$ & $\begin{array}{l}\text { Calibration } \\
\text { error } \\
\text { (Spec. } \\
\text { Grav.) }\end{array}$ & $\begin{array}{l}\text { Square } \\
\text { of cali- } \\
\text { bration } \\
\text { error }\end{array}$ \\
\hline 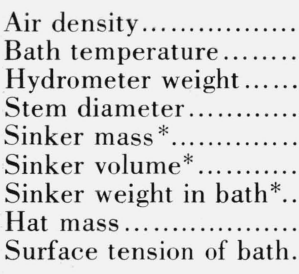 & $\begin{array}{l}1 \mu \mathrm{g} / \mathrm{cm}^{3} \\
0.01{ }^{\circ} \mathrm{C} \\
100 \mu \mathrm{g} \\
127 \times 10^{-6} \mathrm{~cm} \\
30 \mu \mathrm{g} \\
0.0001 \mathrm{~cm}^{3} \\
100 \mu \mathrm{g} \\
10 \mu \mathrm{g} \\
1 \text { dyne } / \mathrm{cm}\end{array}$ & $\begin{array}{l}5 \times 10^{-6} \\
2.3 \times 10^{-6} \\
0 \\
19 \times 10^{-6} \\
3 \times 10^{-6} \\
10 \times 10^{-6} \\
2 \times 10^{-6} \\
.15 \times 10^{-6} \\
35 \times 10^{-6}\end{array}$ & $\begin{array}{l}25 \times 10^{-12} \\
6 \times 10^{-12} \\
0 \\
361 \times 10^{-12} \\
9 \times 10^{-12} \\
100 \times 10^{-12} \\
4 \times 10^{-12} \\
0 \\
1225 \times 10^{-12}\end{array}$ \\
\hline $\begin{array}{l}\text { TOTAL ........ } \\
\text { ESTIMATED } \\
\text { UNCERTAINTY.. }\end{array}$ & & & $\begin{array}{l}1733 \times 10^{-12} \\
42 \times 10^{-6}\end{array}$ \\
\hline
\end{tabular}

*The sinker is the solid-object density standard referred to in the text.

**These uncertainties are assumed to apply to all NBS calibrations made on this type of equipment.

As a matter of interest, figure 5 shows a graph of a hydrometer calibration in S.G. (15.556/15.556 $\left.{ }^{\circ} \mathrm{C}\right)$ with the calibration performed by both the old and new techniques. The four parallel curves are the plot of the table of corrections predicted by the new procedure

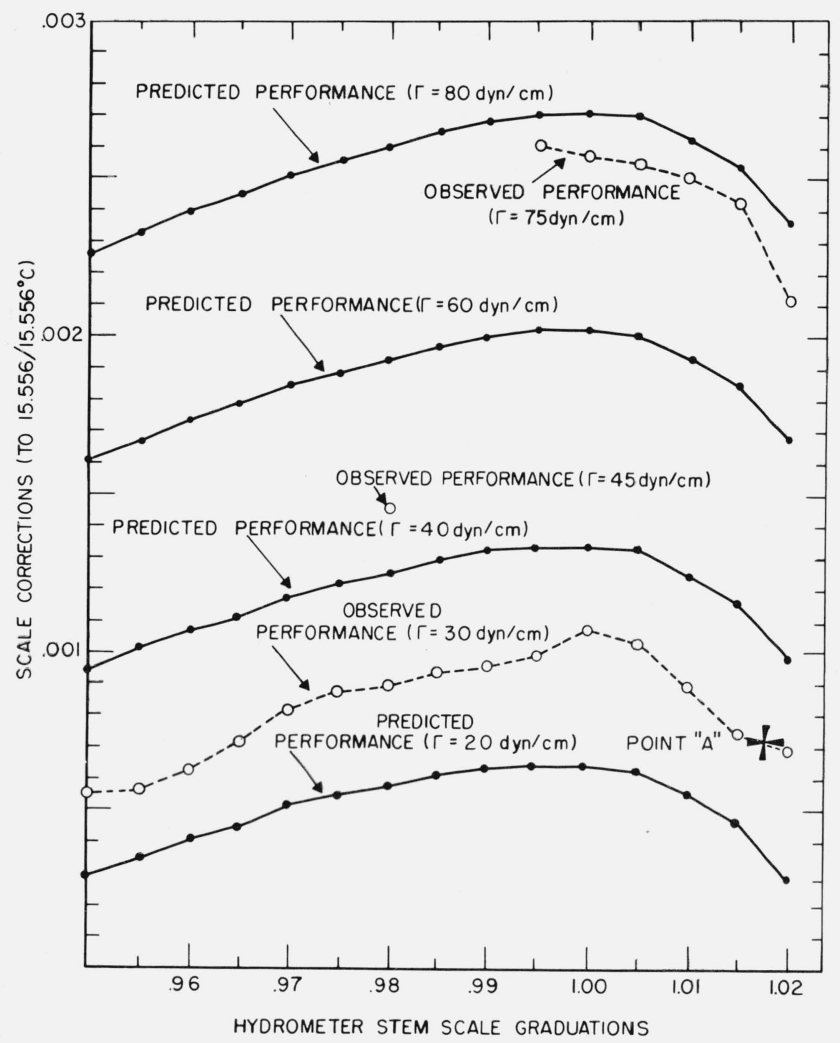

FigURE 5. Hydrometer scale corrections obtained by both the old and new calibration procedure.

The density scale used is $\mathrm{SG}\left(60 / 60^{\circ} \mathrm{F}\right)$. The solid black dots connected by solid lines represent the scale corrections predicted by the computer program at four levels of surface tension. These points are all based upon data taken in the single flotation experiment at point "A," the cross in the lower righthand corner. The open circles represent individual flotation experiments taken in liquids of various surface tensions and densitites using the older calibration procedure. at four levels of surface tension. The data upon which these curves are constructed are based upon a single flotation experiment at point " $A$ " in the lower righthand corner of the graph. In the upper right corner is a sulfuric acid calibration with a surface tension of about 75 dynes per centimeter, and an oil and gasoline calibration is shown at the 30 dynes per centimeter level. A single-point alcohol flotation is indicated at 45 dynes per centimeter. In these classical calibrations, each open circle represents a complete flotation experiment-equal in experimental effort to the flotation experiment at point "A." In other words, in the situation illustrated here, 22 flotation experiments were required for the old calibration (each open circle), but for the new method, only one such experiment was performed (at point "A").

Many Bureau people assisted us in this work. Miss Geraldine Hailes was largely responsible for programming a very awkward data reduction scheme. Roy $\mathrm{H}$. Wampler devised a procedure for converting alcohol densities into other scales. Dr. Daniel P. Johnson checked out the physical validity of the arguments presented.

\section{References}

[1] Rands, J. B., and Bigg, P. H., Hydrometers and hydrometry, NPL Applied Science Note No. 25 (1961).

[2] Bowman, H. A., and Schoonover, R. M., Procedure for high precision hydrostatic weighing, J. Res. NBS 71 C, No. 3 (1967).

\section{Appendix A. Use of a Reference Standard to Calibrate a Working Instrument}

\section{Apparatus}

One glass cylinder approximately seventeen (17) inches long and two (2) inches in diameter; a suitable test liquid.

\section{Test Liquids}

Hydrometers should be calibrated in the liquids in which they are to be used; if this is not possible, a liquid should be selected which has the same, or nearly the same, surface tension. The liquids in the following table are suggested as suitable, although any liquid of proper density and surface tension may be substituted. Intermediate densities may be obtained by mixing:

Liquids Range

Ammonia.

0.62 to 1.00

.85 to 1.00

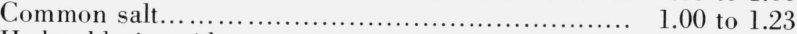

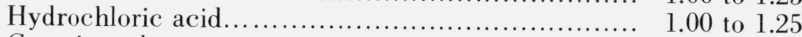

Caustic soda......................................... 1.00 to 1.55

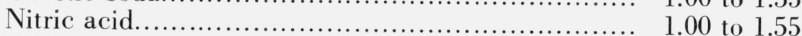

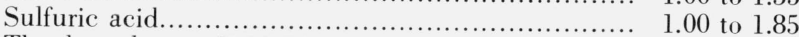

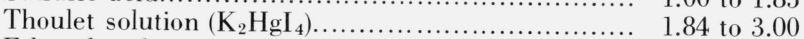

Ethanol and water.................................. 0.750 to 1.000 


\section{Preparation}

(a) Cleanliness.

The accuracy of hydrometer observations depends upon the cleanliness of the instruments and of the liquids in which the observations are made. Extra care should be taken to keep the liquid surface free of contamination.

In order that readings shall be uniform and reproducible, the surface of the hydrometers, and especially of the stem, must be clean, so that the liquid will rise uniformly and merge into an imperceptible film on the stem.

The readiness with which this condition is fulfilled depends somewhat upon the character of the liquid. Certain liquids, such as mineral oils and strong alcoholic mixtures, adhere to the stem very readily. On the other hand, with weak aqueous solutions of sugar, salts, acids, and alcohol, scrupulous cleaning of the stem is required in order to secure the normal condition.

Before being tested, hydrometers may be thoroughly cleaned by dipping in a mixture of one part concentrated sulfuric acid and two parts fuming sulfuric acid, rinsed with water, and dried by wiping with a clean, lint-free cloth; or they may be washed with soap and water, dried, and wiped with a cloth moistened with alcohol to remove any residual soap film. The stems can usually be kept clean during testing by wiping with alcohol (preferably absolute) and drying before each reading.

In many liquids spontaneous changes in surface tension occur due to the formation of surface films of impurities, which may come from the apparatus, the liquid, or the air. In order to avoid the errors due to these changes, the liquid surface could be cleaned with a vacuum hose or the liquid in the cylinder could be caused to overflow just prior to reading the hydrometer.

The necessity for such special manipulation is confined to the reading of hydrometers in liquids that are subject to surface contamination. Such, in general, are aqueous solutions or mixtures of acids, alkalies, salts, sugars, and weak alcohol mixtures. Oils, alcoholic mixtures of strength above 40 percent by volume, and other liquids of relatively low surface tension are not, in general, liable to surface contamination sufficient to cause appreciable changes in hydrometer readings.

\section{(b) Temperature.}

In order that a hydrometer may indicate correctly the density or strength of a specified liquid, it is essential that the liquid be uniform throughout and at the temperature specified on the instrument. If an observation is made at some other temperature than that for which the hydrometer is designed, the reading will be in error. The magnitude of the error will depend upon the thermal expansion of the hydrometer and, in some cases, of the liquid used.

If the latter properties are known, tables of corrections for temperature may be prepared for use with hydrometers at various temperatures. Such tables should be used with caution, and only for approximate results when the temperature differs much from the standard temperature or from the temperature of the surrounding air. (See table 28, NBS Circular C19.)

Publications in which temperature correction tables may be found are listed below.
Ethyl alcohol....

Petroleum oils.

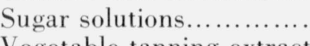

Turpentine.

In comparing two hydrometers having the same standard temperature and made of the same type of glass, the temperature of the liquid need not be considered, since the correction required due to variation from standard temperature is the same for both instruments. But the temperatures of the liquid, the hydrometers, and the surrounding atmosphere should be nearly equal during the observation; otherwise, the temperature of the liquid will be changing, causing differences in density. To insure uniformity in the liquid, thorough mixing is required shortly before making the observation. This may be accomplished with a perforated disk or spiral at the end of a rod long enough to reach the bottom of the cylinder. Motion of this stirrer from top to bottom serves to disperse layers of the liquid of different density.

\section{Test Procedure}

(a) Pour into the cylinder a sufficient amount of the appropriate test liquid to float the hydrometer. Pour the liquid slowly down the side of the cylinder to avoid the formation of air bubbles.

(b) Stir the liquid, avoiding the formation of air bubbles. Make sure the liquid is approximately at room temperature.

(c) Immerse the standard hydrometer in the liquid slightly beyond the point where it floats naturally, then allow it to float freely.

(d) Observe a point slightly below the plane of the liquid surface and then raise the line of vision until this surface, seen as an ellipse, becomes a straight line. The point where this very fine line cuts the hydrometer scale is the reading of the instrument. Holding a white card behind the cylinder just below the liquid level will improve the visibility of the surface.

(e) Immerse the hydrometer to be tested as outlined in item (c), read the hydrometer as outlined in item (d).

(f) Again, read the standard hydrometer as outlined in items (c) and (d).

(g) Average the standard readings and apply scale corrections, supplied by NBS, to the average standard reading.

(h) The difference between the average corrected standard reading and the reading of the hydrometer being tested will be its correction at that point on the scale.

(Paper 73C3\&4-289) 